\title{
CONTAMINACIÓN AMBIENTAL, VARIABILIDAD CLIMÁTICA Y CAMBIO CLIMÁTICO: UNA REVISIÓN DEL IMPACTO EN LA SALUD DE LA POBLACIÓN PERUANA
}

\author{
Gustavo F. Gonzales ${ }^{1,2,3, a}$, Alisson Zevallos ${ }^{2, b}$, Cynthia Gonzales-Castañeda ${ }^{1,2, e}$, Denisse Nuñez ${ }^{2, b}$, \\ Carmen Gastañaga ${ }^{6, c}$, César Cabezas ${ }^{6, d}$ Luke Naeher $^{4, e}$, Karen Levy $^{5, \mathrm{e}}$, \\ Kyle Steenland ${ }^{5, e}$
}

\begin{abstract}
RESUMEN
El presente artículo es una revisión sobre la contaminación del agua, el aire y el efecto del cambio climático en la salud de la población peruana. Uno de los principales contaminantes del aire es el material particulado menor de $2,5 \mu$ (PM 2,5), en la ciudad de Lima, anualmente 2300 muertes prematuras son atribuibles a este contaminante. Otro problema es la contaminación del aire domiciliario por el uso de cocinas con combustible de biomasa, donde la exposición excesiva a PM 2,5 dentro de las casas es responsable de aproximadamente 3000 muertes prematuras anuales entre adultos, con otro número desconocido de muertes entre niños debido a infecciones respiratorias. La contaminación del agua tiene como principales causas los desagües vertidos directamente a los ríos, minerales (arsénico) de varias fuentes, y fallas de las plantas de tratamiento. En el Perú, el cambio climático puede impactar en la frecuencia y severidad del fenómeno de El Niño oscilación del sur (ENSO) que se ha asociado con un incremento en los casos de enfermedades como cólera, malaria y dengue. El cambio climático incrementa la temperatura y puede extender las áreas afectadas por enfermedades transmitidas por vectores, además de tener efecto en la disponibilidad del agua y en la contaminación del aire. En conclusión, el Perú, pasa por una transición de factores de riesgo ambientales, donde coexisten riesgos tradicionales y modernos, y persisten los problemas infecciosos y crónicos, algunos de los cuales se asocian con problemas de contaminación de agua y de aire.
\end{abstract}

Palabras clave: Contaminación del agua; Contaminación del aire; Cambio climático (fuente: DeCS BIREME).

\section{ENVIRONMENTAL POLLUTION, CLIMATE VARIABILITY AND CLIMATE CHANGE: A REVIEW OF HEALTH IMPACTS ON THE PERUVIAN POPULATION}

\begin{abstract}
This article is a review of the pollution of water, air and the effect of climate change on the health of the Peruvian population. A major air pollutant is particulate matter less than $2.5 \mu$ (PM 2.5). In Lima, 2,300 premature deaths annually are attributable to this pollutant. Another problem is household air pollution by using stoves burning biomass fuels, where excessive indoor exposure to PM 2.5 inside the household is responsible for approximately 3,000 annual premature deaths among adults, with another unknown number of deaths among children due to respiratory infections. Water pollution is caused by sewage discharges into rivers, minerals (arsenic) from various sources, and failure of water treatment plants. In Peru, climate change may impact the frequency and severity of EI Niño Southern Oscillation (ENSO), which has been associated with an increase in cases of diseases such as cholera, malaria and dengue. Climate change increases the temperature and can extend the areas affected by vector-borne diseases, have impact on the availability of water and contamination of the air. In conclusion, Peru is going through a transition of environmental risk factors, where traditional and modern risks coexist and infectious and chronic problems remain, some of which are associated with problems of pollution of water and air.
\end{abstract}

Key words: Water pollution; Air pollution; Climate change (source: MeSH NLM).

\footnotetext{
Instituto de Investigaciones de la Altura. Lima, Perú.

Facultad de Ciencias y Filosofía, Universidad Peruana Cayetano Heredia. Lima, Perú.

Academia Nacional de Ciencias. Lima, Perú.

University of Georgia. Georgia, EE. UU.

University of Emory. Georgia. EE. UU.

Instituto Nacional de Salud. Lima, Perú.

a Doctor en Ciencias y doctor en Medicina; ${ }^{\mathrm{b}}$ estudiante de Biología; ${ }^{\mathrm{c}}$ médico cirujano; ${ }^{\mathrm{d}}$ médico infectólogo; ${ }^{\mathrm{e}}$ Philosophal Doctor

Recibido: 10-05-14 Aprobado: 06-08-14
}

Citar como: Gonzales GF, Zevallos A, Gonzales-Castañeda C, Nuñez D, Gastañaga C, Cabezas C, et al. Contaminación ambiental, variabilidad climática y cambio climático: una revisión del impacto en la salud de la población peruana. Rev Peru Med Exp Salud Publica. 2014;31(3):547-56. 


\section{INTRODUCCIÓN}

Muchas enfermedades han aumentado de manera importante en las últimas décadas en los países en vías de desarrollo, particularmente las enfermedades crónicas, las cuales estarían asociadas a contaminantes ambientales (1) que pueden estar en el agua y en el aire.

Por otro lado, la actividad antropogénica está produciendo cambios sin precedentes al medioambiente global. La acumulación de gases de efecto invernadero, como el dióxido de carbono $\left(\mathrm{CO}_{2}\right)$ y el metano, entre otros, tienen implicaciones en el clima del planeta. El mundo se ha calentado en aproximadamente $0,6^{\circ} \mathrm{C}$ en las últimas tres décadas. El tiempo que el $\mathrm{CO}_{2}$ reside en la atmósfera excede los 100 años, por lo cual, lo que hoy se haga en la tierra tendrá repercusiones en las futuras generaciones ${ }^{(2)}$.

Aunque se presume que el cambio climático puede afectar la salud, la evidencia aún es pequeña. Las investigaciones a la fecha se han enfocado a estrés termal, eventos de climas extremos, y enfermedades infecciosas, con alguna atención de estimados futuros sobre producción de alimentos en la región y prevalencia de hambre ${ }^{(3)}$.

En el Perú, un tercio de viviendas utilizan combustible de biomasa para cocinar o para calefacción (4). Los contaminantes producidos por la combustión de esta biomasa tienen efectos negativos en la salud ${ }^{(5,6)}$. El aire exterior en Perú también está contaminado, a un alto nivel comparado con otros países latinoamericanos, según un reporte reciente de la Organización Mundial de la Salud (OMS). Se sabe que la materia particulada en el aire exterior está asociada con varias enfermedades crónicas. El Perú es también un país netamente minero, por lo cual está expuesto a la contaminación ambiental producida por la explotación minera ${ }^{(7)}$; esto implica un importante efecto negativo tanto de la contaminación de aire de exteriores y de interiores.

La diversidad de ecosistemas en el Perú y los grandes desafíos para conservarlos y gestionarlos adecuadamente hacen fundamental la investigación en temas ambientales (8). Para enfocar mejor estos temas, se realizó del 28 al 30 de abril de 2014 en Lima un taller sobre el impacto en salud de la contaminación ambiental de agua, aire y cambio climático, donde los autores participaron. Para profundizar sobre el tema, se realizó una revisión narrativa de la literatura sobre el impacto en la salud que tiene la contaminación del aire y del agua de consumo humano, así como el efecto del cambio climático en el Perú.

\section{CONTAMINACIÓN DEL AGUA}

El Perú es la mayor reserva de América Latina, y se ubica entre los 20 países con mayor disponibilidad de agua del mundo. El $65 \%$ de la población peruana que habita la costa tiene disponibilidad solo del $1,7 \%$ de agua de todo el país. Esto contrasta con la cuenca del Amazonas donde se localiza el 97,7\% del agua, y que está disponible para el $30 \%$ de la población ${ }^{(9)}$. El agua es un constituyente de suma necesidad para la vida, pero también puede ser un agente que daña la salud de las poblaciones, cuando está contaminada con agentes infecciosos o químicos.

\section{AGUA POTABLE Y ENFERMEDAD DIARREICA AGUDA}

En el Perú el acceso al agua potable y saneamiento es aún muy bajo en el ámbito rural a diferencia del espacio urbano; existe una proporción de la población sin acceso a dichos servicios y se sabe que las tasas de cobertura en Perú son menores que en América Latina en general, especialmente en el área rural (Tabla 1). Con el crecimiento poblacional de Lima, la demanda superará largamente la oferta.

En el 2009, nueve de cada diez hogares contaba con agua segura; sin embargo, las brechas entre departamentos como Lima $(98,8 \%)$, Loreto $(62,8 \%)$ y Ucayali $(70,8 \%)$ son notables. Respecto a los hogares con saneamiento básico, es necesario precisar que las diferencias entre el área urbana $(92,9 \%$ ) y el área rural $(67,1 \%)$ también son marcadas ${ }^{(4,10,11)}$. La falta de acceso al agua segura y con un saneamiento básico inadecuado es causal de la prevalencia y persistencia de enfermedades diarreicas agudas (EDA) y las parasitosis ${ }^{(12)}$.

Tabla 1. Proporción de la Población del Perú y otras áreas del mundo con fuente de agua y saneamiento mejorado en el 2011

\begin{tabular}{lccc}
\hline & Total & $\begin{array}{c}\text { Área } \\
\text { urbana }\end{array}$ & $\begin{array}{c}\text { Área } \\
\text { rural }\end{array}$ \\
\hline Fuente de agua mejorada 2011 & & & \\
Perú & $85 \%$ & $91 \%$ & $66 \%$ \\
\hline América Latina y el Caribe & $94 \%$ & $97 \%$ & $82 \%$ \\
\hline Países en desarrollo & $87 \%$ & $97 \%$ & $82 \%$ \\
Mundo & $89 \%$ & $96 \%$ & $81 \%$ \\
\hline Saneamiento mejorado 2011 & & & \\
Perú & $72 \%$ & $81 \%$ & $\mathrm{n} / \mathrm{a}$ \\
\hline América Latina y el Caribe & $82 \%$ & $87 \%$ & $63 \%$ \\
\hline $\begin{array}{l}\text { Países en desarrollo } \\
\text { Mundo }\end{array}$ & $57 \%$ & $74 \%$ & $43 \%$ \\
\hline
\end{tabular}

Fuente: Naciones Unidas, Millenium Development Goals Indicators 
El tener acceso al agua y sistemas de eliminación de excretas por la red pública mejora las conductas sanitarias de las personas, como el lavado de manos antes o luego de una actividad fisiológica, tal como lo muestra un estudio en barrios marginales de Lima, Perú (13). Por otro lado, cuando el acceso al agua potable disminuye, se puede facilitar la contaminación fecal del agua ${ }^{(14,15)}$.

En la región de las Américas, la OMS estima que el rotavirus es responsable de la mayoría de casos de enfermedad diarreica aguda (EDA) en niños < 5 años, seguido por E. coli patógena ${ }^{(16)}$. La EDA también puede verse influenciada por factores de variabilidad climática en poblaciones pobres localizadas en zonas marginales $(17,18)$. Estudios recientes en Perú demuestran que las EDA afectan tanto el peso del niño como su crecimiento (19); igualmente, se ha demostrado en Perú que el acceso al agua y a la eliminación de excretas revierte el efecto adverso sobre el crecimiento de la estatura en niños que previamente tenían inadecuado acceso a agua segura o a eliminación de excretas ${ }^{(20-22)}$.

\section{CONTAMINACIÓN CON ARSÉNICO}

Argentina, Chile y Perú se encuentran expuestos a niveles elevados de arsénico en agua, especialmente de origen subterráneo (23-31). La toxicidad del arsénico depende de la forma en que se encuentre así como su estado de oxidación, siendo los arsénicos inorgánicos solubles más tóxicos que los orgánicos ${ }^{(32)}$. La exposición baja a moderada al arsénico inorgánico ha sido prospectivamente asociada a un aumento en la morbilidad y mortalidad por cáncer ${ }^{(33)}$, enfermedad cardiovascular ${ }^{(34)}$ y diabetes mellitus ${ }^{(35)}$.

En Bangladesh se ha asociado a las enfermedades diarreicas con exposición prenatal de arsénico (36); este elemento puede también aumentar los problemas de diarrea mencionados arriba. En América Latina, con información originada en Argentina, Chile y México, se reportan asociados al arsénico al cáncer de vejiga, de pulmón, de riñón, de hígado y de piel; igualmente, se menciona a las lesiones en la piel, y la enfermedad cardiovascular y respiratoria ${ }^{(24,27)}$. La prevalencia de cada enfermedad es distinta en cada país ${ }^{(37)}$.

En el Perú, la presencia del arsénico en las fuentes de agua para consumo humano se debe tanto a factores naturales de origen geológico como a la explotación minera y refinación de metales por fundición. El mismo proceso geológico de la cordillera de los Andes que se asocia a la presencia natural de arsénico en aguas superficiales y subterráneas, también lo explican los yacimientos de cobre, cuya explotación y posterior fundición contribuye a los niveles de arsénico ambiental.

En el Perú se han reportado niveles de arsénico en agua de consumo humano por encima del límite máximo permisible recomendado en el agua potable de $10 \mathrm{ug} / \mathrm{L}^{(25)}$, que es igual al límite de OMS. Hace poco se publicó un estudio con 111 muestras de agua de consumo en Perú; en doce distritos, de los cuales $86 \%$ suparaban $10 \mathrm{ug} / \mathrm{L}$, y $56 \%$ superaban $50 \mathrm{ug} / \mathrm{L}{ }^{(26)}$. Se ha reportado la presencia de arsénico (180 ug/L) en pozos de Puno, de 200-400 ug/L en el río Locumba, y $25 \mathrm{ug} / \mathrm{L}$ en el agua de consumo en la provincia de Hyatara ${ }^{(27)}$. Igualmente, se ha detectado arsénico en la cuenca del río Rímac, que lleva el agua a Lima, donde en 1994 , el $85 \%$ de la muestras sobrepasaron los $50 \mathrm{ug} / \mathrm{L}^{(27)}$.

Más recientemente, estudios de DIGESA y SEDAPAL encontraron niveles en el río Rímac cerca al $50 \mathrm{ug} / \mathrm{L}$ en los años 1997-2004, salvo los años de 2000, 2001 y 2002, en que los promedios eran de $260 \mathrm{ug} / \mathrm{L}, 710 \mathrm{ug} / \mathrm{L}$, y $780 \mathrm{ug} / \mathrm{L}^{(28)}$. En todo el Perú se ha estimado para el año 2000 que, al menos, 250000 personas bebían agua con concentraciones de arsénico $>50$ ug/L ${ }^{(29-31)}$

Son pocos los estudios de seres humanos en cuanto al arsénico en Perú. Dos estudios de los Centros para el Control y Prevención de Enfermedades de los Estados Unidos (CDC) en regiones mineras de Perú reportaron niveles bajos en la orina, con un promedio de $6 \mathrm{ug} / \mathrm{L}$ en Ancash en la sierra norte ${ }^{(38)}$, y un promedio de $18 \mathrm{ug} / \mathrm{L}$ en la ciudad de Cerro de Pasco en la sierra central ${ }^{(39)}$. El nivel recomendado en la orina es $50 \mathrm{ug} / \mathrm{L}^{(40)}$. En cambio, se ha observado queratosis arsenical en $5,7 \%$ de habitantes de una población residente a los 3290 metros de altitud expuesta a relaves mineros con arsénico ${ }^{(41)}$.

Es importante mencionar que el consumo de arsénico por la población humana no es solamente a través del agua de consumo diario, sino también a través de los alimentos, se estima en América Latina que los alimentos contribuyen hasta el $50 \%$ del total de la ingesta de arsénico ${ }^{(24)}$. Falta mayor información sobre el consumo de arsénico en agua potable en cada región y el impacto en salud, tanto agudo como crónico, de esta exposición.

\section{CONTAMINACIÓN POR PARTICULAS DEL AIRE AMBIENTAL}

La mayoría de los efectos dañinos crónicos de la contaminación del aire ambiental se miden, en la actualidad, a través del material particulado menor a 2,5 micrones (PM 2,5), que penetran los espacios profundos del pulmón ${ }^{(42)}$. En la Figura 1, se nota que 


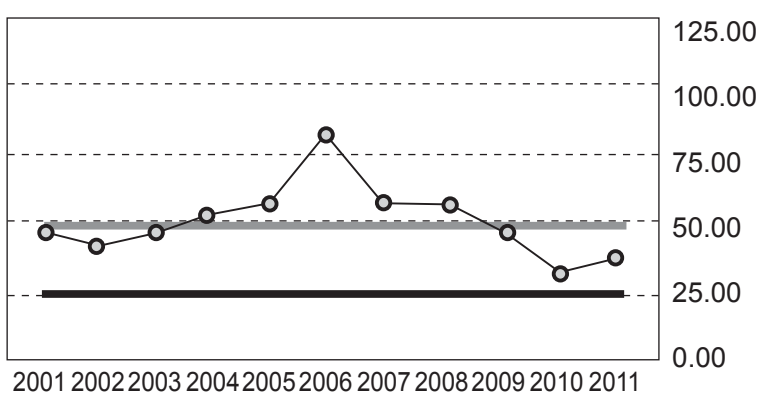

ECA Internacional $=25 \mu \mathrm{g} / \mathrm{m}^{3}$ o PM 2,5 Lima ECA Nacional $=50 \mu \mathrm{g} / \mathrm{m}^{3}$

Figura 1. Concentraciones promedio anuales de materias particuladas menores de 2,5 micras (PM 2,5) en Lima durante el periodo 2001 a 2011.

Fuente: Dirección General de Salud Ambiental (DIGESA), Perú

Elaboración: Observatorio Ambiental de Lima

las partículas PM 2,5 anuales en Lima, la capital del Perú, en el periodo 2001-2011, han tenido un promedio de aproximadamente $50 \mathrm{ug} / \mathrm{m}^{3}$. El nivel de PM 2,5 recomendado por la OMS es $10 \mathrm{ug} / \mathrm{m}^{3}\left(25 \mathrm{ug} / \mathrm{m}^{3}\right.$ en Perú). Para comparación, en Chile el nivel de PM 2,5 en el aire ha bajado de $35 \mathrm{ug} / \mathrm{m}^{3}$ a $25 \mathrm{ug} / \mathrm{m}^{3}$ en el mismo periodo (Comisión Nacional del Medioambiente), cuando en los Estados Unidos ha bajado de $15 \mathrm{ug} / \mathrm{m}^{3}$ a $10 \mathrm{ug} /$ $\mathrm{m}^{3}$ (http://www.epa.gov/airtrends/pm.html). Estos datos indicarían que Lima tiene un problema por resolver con respecto a la contaminación del aire con PM 2,5.

Para medir los posibles efectos en la salud en Lima de los niveles de PM 2,5 mayores a lo recomendado por la OMS, se puede utilizar estudios de cohortes de otros países que consideran los efectos a largo plazo de la contaminación del aire con PM 2,5. En estos estudios se ha comprobado que la exposición crónica al aire ambiental contaminado por partículas resulta en enfermedades crónicas, sobre todo cardiovasculares, cerebrovasculares, y pulmonares, incluyendo cáncer del pulmón y enfermedades pulmonares no malignas. Basado en estos estudios, se ha podido calcular la relación exposición-respuesta entre las partículas PM 2,5 y las enfermedades mencionadas, ello permite la cuantificación del riesgo para la salud de niveles específicos de PM2, $5^{(43,44)}$.

Tomando en cuenta la relación exposición-respuesta descrita por Pope et al. ${ }^{(43)}$ (y notando que $1 \mathrm{ug} / \mathrm{m}^{3} \mathrm{PM} 2,5$ representa una dosis individual de $0,18 \mathrm{mg} \mathrm{PM} \mathrm{2,5/día)} \mathrm{un}$ aumento de $10 \mathrm{ug} / \mathrm{m}^{3}$ (considerado como 'no-expuesto') a $50 \mathrm{ug} / \mathrm{m}^{3}$ (nivel promedio de Lima 2001-2011) corresponde un riesgo relativo de aproximadamente 1,25 tanto para el cáncer pulmonar como para las enfermedades cardiovasculares/cerebrovasculares (ICD9 códigos 401459), o cardiopulmonares (ICD9 códigos 401-459, 460519) (este riesgo relativo concuerda bien con un trabajo reciente $\left.{ }^{(44)}\right)$. Tal riesgo relativo se traduce en una fracción atribuible de $20 \%$ [FA = $(\mathrm{RR}-1) / \mathrm{RR})$ ] para una población donde todos están expuestos, como Lima.

Puesto que el Ministerio de Salud reporta 940 muertes de personas mayores de 20 años por cáncer pulmonar (código ICD10 C34); 9550 muertes por enfermedades cardiovasculares(códigos ICD10 I00-159, I70-199); 1170 muertes por enfermedades cerebrovasculares (ICD10 códigos 160-169) en el Departamento de Lima en 2012 (que corresponden casi todas a la región metropolitana), se puede estimar que hay, aproximadamente, $20 \%$ de estas muertes en Lima, es decir 2330 (190 por cáncer pulmonar, 1910 por problemas cardiovasculares, y 230 por problemas cerebro-vasculares) atribuibles a un nivel aumentado de PM 2,5 en el aire de Lima, encima de $10 \mathrm{ug} / \mathrm{m}^{3}$. A esta cifra se debería agregar las muertes atribuibles a PM 2,5 ambiental en otras ciudades de Perú.

Igualmente, la contaminación del aire en Lima se relaciona directamente con enfermedades respiratorias crónicas en los escolares; así, los escolares de Lima expuestos a un alto tráfico vehicular cerca de casa y/o escuela desarrollan en mayor proporción síntomas de asma y atopía con relación a quienes viven más alejados ${ }^{(45,46)}$. Además, los niños en las zonas rurales tienen menos asma que los niños en zonas urbanas ${ }^{(47)}$.

\section{CONTAMINACION DEL AIRE AMBIENTAL: EL CASO DEL PLOMO}

En el Perú, durante muchos años, la gasolina con plomo era la fuente de emisión más importante para la población urbana ${ }^{(48)}$. En la actualidad, por disposiciones legales, está prohibido el uso del plomo en la gasolina; esto ha tenido un importante impacto, como lo demuestra

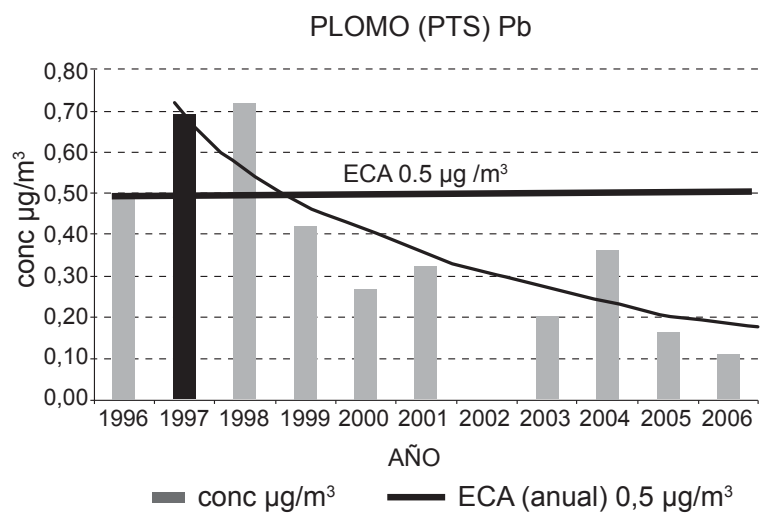

Figura 2. Niveles de plomo medidos en la Estación CONACO en avenida Abancay, Lima. Peridodo: 1996-2006 
el hallazgo de una disminución del plomo en el aire y en la sangre de la población (Figura 2).

Sin embargo, todavía se encuentra en poblaciones locales niveles elevados de plomo, ya sea debido a la actividad minera, a la fundición de metales, desde almacenes de minerales o durante su transporte.

El peligro principal conocido de la exposición al plomo es su efecto sobre el desarrollo intelectual de los niños ${ }^{(49,50)}$. Según el CDC de Atlanta, el nivel de plomo en la sangre de los niños no deben exceder los 10 ug/dL ${ }^{(51)}$. Entre los adultos expuestos a niveles altos, hay evidencia del daño a los riñones ${ }^{(52)}$, de aumento de la presión arterial ${ }^{(53)}$, y un aumento de riesgo de cáncer pulmonar/estómago (54), aunque esto efectos no son considerados tan concluyentes como los efectos en niños.

En lugares donde se almacena el plomo hay gran riesgo de contaminación del aire; así, se ha demostrado niveles altos de plomo en sangre de niños y mujeres posparto en Lima Metropolitana y Callao, siendo mayores en niños que viven en el Callao (26 ug/dL) comparado a 7 ug/dL entre niños en Lima, como una consecuencia de la ubicación de almacenes para el depósito de minerales como el plomo en el Callao ${ }^{(55)}$.

Otros estudios en diversas comunidades de la ciudad minera de Cerro de Pasco (Chaupimarca, Ayapoto y Paragsha) demostraron que el $53 \%$ de niños y aproximadamente el $9 \%$ de mujeres en edad fértil mostraban intoxicación por metales pesados con niveles de plomo en sangre por encima de $10 \mathrm{ug} / \mathrm{dL}{ }^{(51)}$. Igualmente, dos estudios comparativos entre sujetos varones adultos de Lima y Cerro de Pasco muestran mayores niveles de plomo en sangre en varones de la ciudad de Cerro de Pasco, distribuidos entre mineros y no mineros ${ }^{(56,57)}$.

Los niveles de plomo en suelo que exceden $1200 \mathrm{mg} / \mathrm{kg}$, un estándar de referencia para suelo de residencias, se encuentran presentes en 35 de 74 sitios en Cerro de Pasco comparados con 4 de 47 sitios evaluados alrededor de operaciones nuevas cerca de Huaral, en el norte de Lima ${ }^{(58)}$. En la ciudad de La Oroya, que desde el año 1922 la población se encuentra expuesta a las emisiones de la planta de fundición, se ha observado en las gestantes tasa altas de plomo ( $\geq 10 \mathrm{ug} / \mathrm{dL})$ en el $71,4 \%$ en La Oroya Nueva, $100 \%$ en La Oroya vieja y $82,5 \%$ en otras zonas. Igualmente, las tasas de plomo alto en el cordón umbilical oscilan entre 52,4 y $88,9 \%{ }^{(59)}$.

Todavía faltan estudios en Perú documentando efectos dañinos en el desarrollo intelectual de los niños de estas zonas, o documentando efectos crónicos entre adultos.

\section{CONTAMINACIÓN DE INTERIORES: EL CASO DEL COMBUSTIBLE DE BIOMASA}

La contaminación del aire intradomiciliario se ha vinculado con enfermedades respiratorias entre niños ${ }^{(60)}$, y resultados reproductivos adversos ${ }^{(61)}$. Se estima que en 2010 el aire intradomiciliario fue responsable de 3,5 millones de muertes prematuras en el mundo, que es mayor al número de muertes atribuibles a contaminación ambiental del aire $\left(3,1\right.$ millones de muertes) ${ }^{(42)}$. Estas muertes se deben a enfermedades respiratorias entre niños (aproximadamente $40 \%$ ), y muertes por enfermedades cardiopulmonares y cardiovasculares entre adultos (60\%).

En Perú, estudios relacionados a la contaminación intradomiciliaria se han llevado a cabo midiendo principalmente los niveles de monóxido de carbono (CO) y PM 2,5 en el aire ${ }^{(62-64)}$, y a veces, biomarcadores como 8-hidroxi-2'-deoxiguanosina y 8-isoprostano en la orina ${ }^{(65)}$. Algunas publicaciones muestran resultados que tratan de medir el impacto de instalar cocinas mejoradas, que presenta varios desafíos ${ }^{(66)}$. Con los datos limitados en Perú, que se tiene acceso hasta ahora, parece que las cocinas mejoradas pueden bajar los niveles de CO y PM 2,5 inmediatamente en un porcentaje importante $(47-74 \%){ }^{(67)}$, pero con el tiempo esta reducción parece ser menor (25-50\%), probablemente, entre otros, a falta de mantenimiento de las cocinas ${ }^{(68)}$. El nivel promedio de PM 2,5 de personas viviendo en hogares usando biomasa, puede ser aproximadamente $100 \mathrm{ug} / \mathrm{m}^{3}$ de PM 2,5, tanto en las ciudades ${ }^{(69)}$ como en la zona rural ${ }^{(67)}$, usando mediciones de muestras personales. Dado eso, una reducción de 25-50\% (a 50-75 ug/ $/ \mathrm{m}^{3}$ ) con cocinas mejoradas no será suficiente para eliminar el riesgo excesivo de enfermedades; para ello, tendría que llegar a un nivel de $10 \mathrm{ug} / \mathrm{m}^{3} \mathrm{PM}$ 2,5, tal como lo recomienda la OMS.

En la Figura 3 se presentan los porcentajes de viviendas que utilizan solo leña o solo gas en el Perú entre los años 2003 y 2012. A nivel nacional se observa una disminución en el porcentaje de hogares que usan solo leña, de algo menos del $30 \%$ en el 2003 a un poco más del $15 \%$ en el 2012 ; por su parte, hay un incremento en el mismo período de los hogares que usan solo gas (40\% en 2012). Hay muchos hogares que usan ambos. Si en el Perú existen 6 millones de viviendas, los datos indicarían que 3,6 millones de viviendas podrían estar usando de alguna manera combustible de biomasa.

En Perú, según estimaciones de OMS, cerca de 10 millones de habitantes están expuestos a combustible de biomasa (leña, champa, bosta, carbón) para cocinar ${ }^{(67)}$. El nivel promedio estimado de PM 2,5 de personas viviendo en estos hogares, sería aproximadamente $100 \mathrm{ug} / \mathrm{m}^{3} \mathrm{PM}$ $2,5^{(62,64)}$. Este nivel es dos veces el nivel de PM 2,5 en 


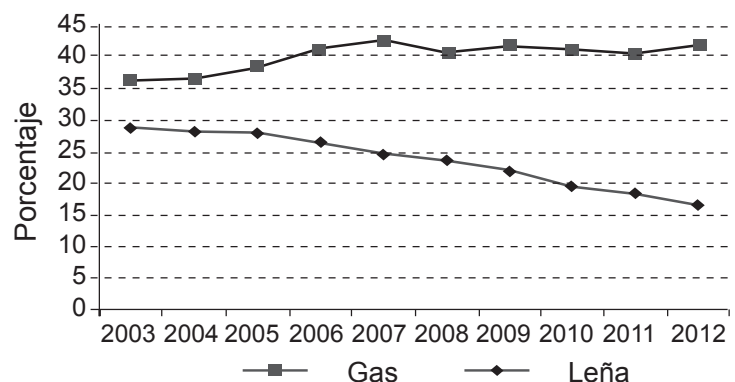

Figura 3. Porcentaje de hogares en el Perú que usan solo leña o solo gas para cocinar: Evolución entre 2003 y 2012.

Fuente: Instituto Nacional de Estadística e Informática.

el aire de Lima. Siguiendo la lógica de los cálculos arriba relacionados con el aire ambiental, el riesgo relativo para la muerte cardiovascular/cardiopulmonar sería aproximadamente 1,3-1,4 debido a esta exposición comparada con la recomendada por la OMS de $10 \mathrm{ug} / \mathrm{m}^{3}$, y la fracción de muertes atribuibles a PM 2,5 debería ser aproximadamente $25 \%[(1,35-1) / 1,35]$. Asumiendo que los 10 millones de habitantes que usan biomasa tienen aproximadamente las mismas tasas de mortalidad que los habitantes de Lima, podemos estimar que habría aproximadamente 3000 muertes prematuras por causas cardiopulmonares anuales entre adultos, por exposición excesiva a la PM 2,5. A ese número habría que agregarse otra cantidad desconocida de muertes prematuras entre niños por enfermedades respiratorias, principalmente la neumonía.

La exposición crónica a combustible de biomasa se asocia con un aumento en el grosor de la íntima-media de la arteria carótida y mayor prevalencia de la placa ateroesclerótica e hipertensión arterial ${ }^{(68)}$. Esto genera un alto riesgo cardiovascular. El cocinar con combustible de biomasa y vivir en grandes altitudes puede tener un efecto adicional adverso sobre la salud materno-perinatal, teniendo en cuenta que la altitud se asocia a menor peso al nacer, por la hipoxia ambiental ${ }^{(69)}$. En un estudio caso-control en dos zonas altoandinas en Perú (Apurímac y Huancavelica) se encuentra que $52 \%$ de madres de niños nacidos con bajo peso usaban combustible de biomasa para cocinar, en comparación con las madres con niños normales quienes en un $30 \%$ usaron combustible de biomasa ${ }^{(5)}$.

\section{CAMBIO CLIMÁTICO}

El cambio climático, causado por seres humanos, afecta a todas las naciones del mundo ${ }^{(70)}$. Este cambio está asociado con un aumento de temperatura y más variabilidad climática. Muchas de las enfermedades prevalentes en Perú pueden ser afectadas por fluctuaciones climáticas, desde la mortalidad cardiovascular y enfermedades respiratorias debido a las ondas de calor o una alterada trasmisión de enfermedades infecciosas y a la malnutrición debido a escasez de alimentos por falta de agua.

\section{AUMENTO DE TEMPERATURA Y LA EXPANSIÓN DEL ÁREA DE LOS VECTORES}

Un impacto importante del cambio climático es que la trasmisión de enfermedades infecciosas, antaño caracterizadas en una zona, ahora se puede ver en otras, por el cambio del hábitat más adecuado de los vectores. Por ejemplo, en la actualidad se han reportado cambios altitudinales de la presencia de vectores de la leishmaniosis cutánea en Colombia y Perú (71). En América del Sur, el paludismo, la leishmaniosis, el dengue, la enfermedad de Chagas y la esquistosomiasis son las principales enfermedades de transmisión vectorial sensibles al clima ${ }^{(72)}$.

Recientemente se ha documentado la infestación urbana del vector de la enfermedad de Chagas en Arequipa, Perú, y entre otras variables que pueden explicar esta presencia se encuentra el aumento de la temperatura de la superficie terrestre ${ }^{(73,74)}$.

\section{VARIABILIDAD CLIMÁTICA, EL NIÑO, Y EFECTOS EN LA SALUD}

El fenómeno de El Niño oscilación sur (ENSO) es la alteración climática anual que se manifiesta de modo cíclico aproximadamente cada 4 años en relación a la corriente de El Niño, y que puede variar de intensidad de leve, moderada o severa. El fenómeno de El Niño se manifiesta con lluvias intensas en la zona desértica de la costa norte con graves sequías en la región sur, particularmente altoandina, elevación de la temperatura del mar y disminución de la temperatura en la selva ${ }^{(75,76)}$.

Es de destacar que de 10 meganiños registrados desde el siglo XVI en el Perú, dos ocurrieron en las últimas décadas 1982/1983 y 1997/1998. La previa a ellas fue en 1925. Queda aún por dilucidar cuánto de esto se asocia al cambio climático antropogénico que se describe para el planeta.

Se puede estudiar los efectos a la salud del ENSO en Perú para entender cómo los cambios en el clima, que ocurran en el futuro, pueden afectar a la salud humana ${ }^{(18)}$. De esta manera, la experiencia obtenida en Perú puede servir a otros países del mundo para entender los cambios que se puede esperar cuando se presenta una mayor variabilidad en el clima.

En la epidemia del cólera se ha planteado que ocurre una proliferación autóctona de $V$. cholera asociada al aumento de la temperatura por efecto del fenómeno de El Niño. Se indica que una vez introducidas en las comunidades costeras en concentraciones suficientes para la infección humana, el cólera se expandió a través del agua y por alimentos contaminados ${ }^{(77)}$. Basado en el número de 
casos de cólera reportados en Perú de 1997 al 2000, se encontró una correlación significativa entre la incidencia de cólera y una elevación de la temperatura de la superficie del mar en toda la costa peruana. Igualmente, se muestra que tanto el agua del mar de la costa y el zooplancton actúan como reservorios para el cólera en el Perú (78).

Datos epidemiológicos e investigaciones de laboratorio mostraron que las infecciones con $V$. parahemolíticus observados en la costa del Perú se asocian con el episodio de El Niño 1997. Este patrón fue similar al previamente reportado para el inicio de la epidemia del cólera en 1991 (79). A pesar de estos datos, son necesarias mayores investigaciones para demostrar fehacientemente esta asociación entre el cólera y El Niño.

Aparte del cólera, hay índices de que El Niño está relacionado con la diarrea. Durante los años 19971998, El Niño aumentó la temperatura ambiente de Lima en $5{ }^{\circ} \mathrm{C}$ por encima de lo normal, y el número de admisiones diarias por diarrea aumentaron en $200 \%$ de la tasa previa. Se atribuyeron a El Niño los 6225 casos del exceso de admisiones, ello significó un costo de USD 277 000. Durante el periodo antes de El Niño, las admisiones por diarrea aumentaron en $8 \%$ por $1{ }^{\circ} \mathrm{C}$ de aumento de la temperatura ambiental ${ }^{(18)}$. La tasa de diarrea en primavera aumentó en 55\% durante EI Niño, comparado con datos antes de El Niño ${ }^{(17)}$.

Otra epidemia asociada al clima es la del dengue. El clima influencia la ecología del dengue al afectar la dinámica del vector (Aedes aegypti), el desarrollo del agente, y/o las interacciones entre el humano y el mosquito ${ }^{(80)}$. En los últimos años ha ocurrido una expansión global del dengue y las causas parecen ser multifactoriales pero aún poco entendidas.

En países influenciados por la variabilidad climática ENSO y donde se registra aumento de la temperatura y de las precipitaciones, como el reportado para Matamoros, en la frontera de México y Texas, la incidencia del dengue aumenta en 2,6\% (IC 95\%: $0,2-5,1)$ una semana después que aumenta $1{ }^{\circ} \mathrm{C}$ de temperatura ${ }^{(81)}$. En el Perú, en 1958 el Aedes aegypti fue erradicado de todo el país; sin embargo, en octubre de 1984 el mosquito reaparece en Iquitos ${ }^{(82)}$. Hacia el 2005 se han presentado casos de dengue en más de trece departamentos, incluyendo Lima ${ }^{(83)}$.

\section{CONCLUSIONES}

El Perú, a pesar de su desarrollo económico, aún pasa por un periodo de transición de factores de riesgo ambientales, ya que coexisten tanto riesgos tradicionales como modernos, donde persisten los problemas infecciosos asociados con problemas crónicos, algunos de los cuales se asocian a problemas de contaminación de agua y del aire.

Por otro lado, el Perú está sometido a la variabilidad climática producto del fenómeno de El Niño estando en la actualidad inmerso en mayores frecuencias y severidad de su presentación, y por la probable extensión del área afectada por vectores de enfermedades infecciosas. En cuanto al cambio climático, se hace énfasis en la amplia y valiosa información obtenida en Perú, y que se puede emplear en otros contextos. Queda, sin embargo, mucho por analizar sobre la implicancia del cambio climático en la salud. Los datos existentes permiten sugerir una posibilidad de asociación entre El Niño y el cambio climático, pero se requiere más investigación para comprobarla.

Esta revisión ha permitido detectar la necesidad de mayor información que, si bien puede existir, no está al acceso o en publicaciones para su análisis. Hay una urgencia imperiosa de estudios sobre el impacto de la contaminación de arsénico en agua sobre la salud. Igualmente, permite reflejar la situación del Perú en su salud ambiental donde se detectan problemas en acceso a agua segura y a liberación de excretas, e importantes fuentes de contaminación de aire en interiores y exteriores que deben merecer la atención de las autoridades de salud del país. Un área de investigación importante sería cuantificar el impacto a largo plazo del uso de cocinas mejoradas en la salud.

Se hace necesario profundizar en el estudio del impacto en la salud de la población peruana que tiene la contaminación del aire, del agua, y de la mayor frecuencia y severidad de la variabilidad climática influenciada por el cambio climático antropogénico.

Contribuciones de autoría: GGR y KS participaron en la concepción y diseño del artículo, GGR, AZC, CGC, DNS, MCG, CCS, LN, KL, KS participaron en el análisis e interpretación de datos, redacción del artículo, revisión crítica del artículo y aprobación de la versión final, KS participó en la obtención de financiamiento.

Fuente de financiamiento: Grant 5R24TW009545 de los Institutos Nacionales de salud (NIH) de los Estados Unidos y del proyecto Especial Promoción de la Ciencia y de la Tecnología en el Perú financiado a la Academia Nacional de Ciencias por el Consejo Nacional de Ciencia, tecnología e Innovación (CONCYTEC).

Conflictos de interés: los autores declaran no tener conflictos de interés. 


\section{REFERENCIAS BIBLIOGRÁFICAS}

1. Gall ET, Carter EM, Earnest CM, Stephens B. Indoor air pollution in developing countries: research and implementation needs for improvements in global public health. Am J Public Health. 2013 Apr;103(4):e67-72. doi: 10.2105/AJPH.2012.300955.

2. Haines A, Patz JA. Health effects of climate change. JAMA. 2004 Jan 7:291(1):99-103.

3. McMichael AJ, Woodruff RE, Hales S. Climate change and human health: present and future risks. Lancet. 2006 Mar 11;367(9513):859-69.

4. Instituto Nacional de Estadística e Informática (INEI). Censos Nacionales 2007: XI de Población y VI de Vivienda. Perfil Sociodemográfico del Perú. 2da ed. Lima: INEI; 2008.

5. Yucra S, Tapia V, Steenland K, Naeher LP, Gonzales GF. Association between biofuel exposure and adverse birth outcomes at high altitudes in Peru: a matched case-control study. Int J Occup Environ Health. 2011 OctDec;17(4):307-13.

6. Sukhsohale ND, Narlawar UW, Phatak MS. Indoor air pollution from biomass combustion and its adverse health effects in central India: an exposure-response study. Indian J Community Med. 2013 Jul;38(3):162-7. doi: 10.4103/09700218.116353 .

7. Astete J, Cáceres W, Gastañaga M, Lucero M, Sabastizagal I, Oblitas T, et al. Intoxicación por plomo y otros problemas de salud en niños de poblaciones aledañas a relaves mineros. Rev Peru Med Exp Salud Publica. 2009 Ene-Mar;26(1):15-9.

8. Perú, Ministerio del Ambiente (MINAN). Agenda de investigación ambiental. Lima: MINAM; 2013.

9. Autoridad Nacional del Agua (ANA). Política y Estrategia Nacional de Recursos Hídricos del Perú. Lima: ANA; 2009.

10. Yang H, Bain R, Bartram J, Gundry S, Pedley S, Wright J. Water safety and inequality in access to drinkingwater between rich and poor households. Environ Sci Technol. 2013 Feb 5;47(3):1222-30. doi: 10.1021/ es303345p.

11. Oswald WE, Hunter GC, Kramer MR, Leontsini E, Cabrera L, Lescano $\mathrm{AG}$, et al. Provision of private, piped water and sewerage connections and directly observed handwashing of mothers in a peri-urban community of Lima, Peru. Trop Med Int Health. 2014 Apr;19(4):388-97. doi: 10.1111/ tmi.12262.

12. Gibson KE. Viral pathogens in water: occurrence, public health impact, and available control strategies. Curr Opin Virol. 2014 Feb;4:50-7. doi: 10.1016/j. coviro.2013.12.005.

13. Oswald WE, Hunter GC, Kramer MR, Leontsini E, Cabrera L, Lescano AG, et al. Provision of private, piped water and sewerage connections and directly observed handwashing of mothers in a peri-urban community of Lima, Peru. Trop Med Int Health. 2014 Apr;19(4):388-97. doi: 10.1111/ tmi.12262.

14. Oswald WE, Lescano AG, Bern C, Calderon MM, Cabrera L, Gilman RH. Fecal contamination of drinking water within peri-urban households, Lima, Peru. Am J Trop Med Hyg. 2007 Oct;77(4):699-704

15. Oswald WE, Hunter GC, Lescano AG, Cabrera L, Leontsini E, Pan WK, et al. Direct observation of hygiene in a Peruvian shantytown: not enough handwashing and too little water. Trop Med Int Health. 2008 Nov;13(11):1421-8. doi: 10.1111/j.1365-3156.2008.02177.x.

16. Lanata CF, Fischer-Walker CL, Olascoaga AC, Torres CX, Aryee MJ, Black RE; Child Health Epidemiology Reference Group of the World Health Organization and UNICEF. Global causes of diarrheal disease mortality in children $<5$ years of age: a systematic review. PLoS One. 2013 Sep 4;8(9):e72788.

17. Bennett A, Epstein LD, Gilman RH, Cama V, Bern C, Cabrera L, et al. Effects of the 1997-1998 El Niño episode on community rates of diarrhea. Am J Public Health. 2012 Jul;102(7):e63-9. doi: 10.2105/AJPH.2011.300573.

18. Checkley W, Epstein LD, Gilman RH, Figueroa D, Cama RI, PatzJA, et al.Effects of EI Niño and ambient temperature on hospital admissions for diarrhoeal diseases in Peruvian children. Lancet. 2000 Feb 5;355(9202):442-50.

19. Richard SA, Black RE, Gilman RH, Guerrant RL, Kang G, Lanata CF, et al. Diarrhea in early childhood: short-term association with weight and long-term association with length. Am J Epidemiol. 2013 Oct 1;178(7):1129-38. doi: $10.1093 /$ aje/kwt094.
20. Checkley W, Gilman RH, Black RE Epstein LD, Cabrera L, Sterling CR, et al. Effect of water and sanitation on childhood health in a poor Peruvian peri-urban community. Lancet. 2004 Jan 10;363(9403):112-8.

21. Checkley W, Epstein LD, Gilman RH, Cabrera L, Black RE. Effects of acute diarrhea on linear growth in Peruvian children. Am J Epidemiol. 2003 Jan 15;157(2):166-75.

22. Guerrant RL, DeBoer MD, Moore SR, Scharf RJ, Lima AA. The impoverished gut--a triple burden of diarrhoea, stunting and chronicdisease.Nat Rev Gastroenterol Hepatol. 2013 Apr;10(4):220-9. doi: 10.1038/nrgastro.2012.239.

23. Bundschuh J, Litter MI, Parvez F, Román-Ross G, Nicolli HB, Jean $\mathrm{JS}$, et al. One century of arsenic exposure in Latin America: a review of history and occurrence from 14 countries. Sci Total Environ. 2012 Jul 1;429:2-35. doi: 10.1016/j.scitotenv.2011.06.024.

24. McClintock TR, Chen Y, Bundschuh J, OliverJT, NavoniJ, Olmos V, et al. Arsenic exposure in Latin America. Biomarkers, risk assessments and related health effects. Sci Total Environ. 2012 Jul 1;429:76-91. doi: 10.1016/j.scitotenv.2011.08.051.

25. Ministerio de Ambiente. Estándares nacionales de calidad ambiental para agua. DS N002-2008-MINAM, Perú. El Peruano, 2008:377222-377227.

26. George CM, Sima L, Arias $\mathrm{MH}$, Mihalic J, Cabrera LZ, Danz D, et al. Arsenic exposure in drinking water: an unrecognized health threat in Peru. Bull World Health Organ. 2014 Aug 1;92(8):565-72. doi: 10.2471/ BLT.13.128496.

27. Castro de Esparza ML. Arsénico en el agua de bebida de América Latina y su efecto en la salud pública. Hojas de Divulgación Técnica. Hoja de divulgación técnica No95. Lima: OPS; 2004.

28. Juarez HS. Contaminación del río Rímac por metales pesados y efecto en la agricultura en el Cono Este de Lima Metropolitana. Tesis para obtener el grado de Magister en Ciencias Ambientales. Universidad Nacional Agraria La Molina: Lima, Perú. 2006.

29. Sancha AM, Castro de Esparza ML. Arsenic status and handling in Latin America. Lima: AIDIS/DIAGUA, CEPIS/OPS; 2000 
30. Castro de Esparza ML. Arsénico en el agua de bebida de la población de los valles de Locumba y Chipe. Lima: OPS/ CEPIS; 2002.

31. Castro deEsparzaML. Mejoramiento dela calidad del agua de pozos en zonas rurales de Puno, Perú. En: Litter MI, Sancha AM, Ingallinella AM, editors. Tecnologías económicas para el abatimiento de arsénico en aguas. Buenos Aires: Editorial Programa Iberoamericano de Ciencia y Tecnología para el Desarrollo; 2010. p. $243-56$.

32. Mandal BK, Suzuki KT. Arsenic round the world: a review. Talanta. 2002 Aug $16 ; 58(1): 201-35$.

33. García-Esquinas E, Pollán M, Umans JG, Francesconi KA, Goessler W, Guallar E, et al. Arsenic exposure and cancer mortality in a US-based prospective cohort: the strong heart study. Cancer Epidemiol Biomarkers Prev. 2013 Nov;22(11):1944-53. doi: 10.1158/1055-9965.EPI-13-0234-T.

34. Moon KA, Guallar E, Umans JG, Devereux RB, Best LG, Francesconi $\mathrm{KA}$, et al. Association between exposure to low to moderate arsenic levels and incident cardiovascular disease. A prospective cohort study. Ann Intern Med. 2013 Nov 19;159(10):649-59.

35. Kuo CC, Moon K, Thayer KA, NavasAcien A. Environmental chemicals and type 2 diabetes: an updated systematic review of the epidemiologic evidence. Curr Diab Rep. 2013 Dec;13(6):831-49. doi: 10.1007/s11892-013-0432-6.

36. Rahman A, Vahter M, Ekström EC, Persson LÅ. Arsenic exposure in pregnancy increases the risk of lower respiratory tract infection and diarrhea during infancy in Bangladesh. Environ Health Perspect. 2011 May;119(5):71924. doi: 10.1289/ehp.1002265.

37. Naujokas MF, Anderson B, Ahsan $\mathrm{H}$, Aposhian HV, Graziano $\mathrm{JH}$, Thompson C, et al. The broad scope of health effects from chronic arsenic exposure: update on a worldwide public health problem. Environ Health Perspect. 2013 Mar;121(3):295-302. doi: 10.1289/ehp.1205875.

38. Yip F, Azziz-Baumgartner, Luber G, Miller G, Patel M, Schier J. Heavy metal levels among people living in Communities on the Ayash River Basin: Huari, April 4-14, 2006, Report from Centers for Disease Control, Atlanta, Ga, USA, Feb 21, 2007.
39. Conklin L, Sánchez CA, Neri A, Staley P, Blumenthal W, Jarrett JM, et al. Reporte Final: Exposiciones a metales pesados en niños y mujeres en edad fértil en tres comunidades mineras Cerro de Pasco, Perú. 21 de Mayo - 4 de Julio de 2007. Lima: Department of Health \& Human Services-Centers for Disease Control and Prevention; 2008.

40. Ministerio de Salud. Guía técnica: Guía de práctica clínica para el diagnóstico y tratamiento de la intoxicación por arsénico. Lima: MINSA; 2011. p. 3-19.

41. Ramos W, Galarza C, Ronceros G, de Amat F, Teran M, Pichardo L, et al. Noninfectious dermatological diseases associated with chronic exposure to mine tailings in a Peruvian district. $\mathrm{Br} \mathrm{J}$ Dermatol. 2008 Jul;159(1):169-74. doi: 10.1111/j.1365-2133.2008.08630.x.

42. Lim SS, Vos T, Flaxman AD, Danaei G, Shibuya K, Adair-Rohani H, et al. A comparative risk assessment of burden of disease and injury attributable to 67 risk factors and risk factor clusters in 21 regions, 1990-2010: a systematic analysis for the Global Burden of Disease Study 2010. Lancet. 2012 Dec 15;380(9859):2224-60. doi: 10.1016/ S0140-6736(12)61766-8.

43. Pope CA 3rd, Burnett RT, Turner MC, Cohen A, Krewski D, Jerrett M, et al. Lung cancer and cardiovascular disease mortality associated with ambient air pollution and cigarette smoke: shape of the exposureresponse relationships. Environ Health Perspect. 2011 Nov;119(11):1616-21. doi: 10.1289/ehp.1103639.

44. Burnett RT, Pope CA 3rd, Ezzati M, Olives C, Lim SS, Mehta S, et al. An integrated risk function for estimating the global burden of disease attributable to ambient fine particulate matter exposure. Environ Health Perspect. 2014 Apr;122(4):397-403. doi: 10.1289/ ehp.1307049.

45. Carbajal-Arroyo L, Barraza-Villarreal A, Durand-Pardo R, Moreno-Macías H, Espinoza-Lain R, Chiarella-Ortigoda $\mathrm{P}$, et al. Impact of traffic flow on the asthma prevalence among school children in Lima, Peru. J. Asthma. 2007 Apr;44(3):197-202.

46. Baumann LM, Robinson CL, Combe JM, Gomez A, Romero K, Gilman $\mathrm{RH}$, et al. Effects of distance from a heavily transited avenue on asthma and atopy in a periurban shantytown in Lima,
Peru. J Allergy Clin Immunol. 2011 Apr;127(4):875-82. doi: 10.1016/j. jaci.2010.11.031.

47. Robinson CL, Baumann LM, Romero K, Combe JM, Gomez A, Gilman RH, et al. Effect of urbanisation on asthma, allergy and airways inflammation in a developing country setting. Thorax. 2011 Dec;66(12):1051-7. doi: 10.1136/ thx.2011.158956.

48. Naeher LP, Aguilar-Villalobos M, Miller T. Blood lead survey of children, pregnant women, professional drivers, street workers, and office workers in Trujillo, Peru. Arch Environ Health. 2004 Jul;59(7):359-62.

49. Liu J, Lewis G. Environmental toxicity and poor cognitive outcomes in children and adults. J Environ Health. 2014 JanFeb;76(6):130-8.

50. Vega-Dienstmaier JM, SalinasPiélago JE, Gutiérrez-Campos M del $\mathrm{R}$, Mandamiento-Ayquipa RD, YaraHokama M del C, Ponce-Canchihuamán $\mathrm{J}$, et al. Lead levels and cognitive abilities in Peruvian children. Rev Bras Psiquiatr. 2006 Mar;28(1):33-9.

51. Ramos W, Munive L, Alfaro M, Calderón M, Gonzáles I, Núñez Y. Intoxicación plúmbica crónica: una revisión de la problemática en el Perú. Rev Peru Epidemiol. 2009; agosto;13(2):1-8.

52. Ekong EB, Jaar BG, Weaver VM. Leadrelated nephrotoxicity: a review of the epidemiologic evidence. Kidney Int. 2006 Dec;70(12):2074-84.

53. Nawrot TS, Thijs L, Den Hond EM, Roels HA, Staessen JA. An epidemiological reappraisal of the association between blood pressure and blood lead: a meta-analysis. J Hum Hypertens 2002 Feb;16(2):123-31.

54. International Agency for Research on Cancer. Inorganic and organic lead compounds. (IARC Monographs on the Evaluation of the Carcinogenic Risks to Humans). 2006;87:1-471.

55. Espinoza R, Hernández-Avila M, Narciso J, Castañaga C, Moscoso S, Ortiz G, et al. Determinants of blood-lead levels in children in Callao and Lima metropolitan area. Salud Publica Mex. 2003;45 Suppl 2:S209-19.

56. Gonzales GF, Gasco M, Tapia V, GonzalesCastañeda C. High serum testosterone levels are associated with excessive erythrocytosis of chronic mountain sickness in men. Am J Physiol Endocrinol Metab. 2009 Jun;296(6):E1319-25. doi: 10.1152/ajpendo.90940.2008. 
57. Gonzales GF, Lozano-Hernández R, Gasco M, Gonzales-Castañeda C, Tapia $\mathrm{V}$. Resistance of sperm motility to serum testosterone in men with excessive erythrocytosis at high altitude. Horm Metab Res. 2012 Dec;44(13):987-92. doi: $10.1055 / \mathrm{s}-0032-1321854$.

58. van Geen A, Bravo C, Gil V, Sherpa S, Jack D. Lead exposure from soil in Peruvian mining towns: a national assessment supported by two contrasting examples. Bull World Health Organ. 2012 Dec 1;90(12):878-86. doi: 10.2471/ BLT.12.106419.

59. Castro-Bedriñana J, Chirinos-Peinado D, Ríos-Ríos E. Niveles de plomo en gestantes y neonatos en la ciudad de La Oroya, Perú. Rev. Peru. Med. Exp. Salud Pública. 2013 Jul;30(3):393-8.

60. Brugha R, Grigg J. Urban air pollution and respiratory infections. Paediatr Respir Rev. 2014 Jun;15(2):194-9. doi: 10.1016/j.prrv.2014.03.001.

61. Pope DP, Mishra V, Thompson L, Siddiqui AR, Rehfuess EA, Weber M, et al. Risk of low birth weight and stillbirth associated with indoor air pollution from solid fuel use in developing countries. Epidemiol Rev 2010 Apr;32(1):70-81. doi: $10.1093 /$ epirev/mxq005.

62. Li Z, Sjödin A, Romanoff LC, Horton K, Fitzgerald CL, Eppler A, et al. Evaluation of exposure reduction to indoor air pollution in stove intervention projects in peru by urinary biomonitoring of polycyclic aromatic hydrocarbon metabolites. Environ Int. 2011 Oct;37(7):1157-63. doi: 10.1016/j. envint.2011.03.024.

63. Hartinger SM, Commodore AA, Hattendorf J, Lanata CF, Gil AI, Verastegui H, et al. Chimney stoves modestly improved indoor air quality measurements compared with traditional open fire stoves: results from a small-scale intervention study in rural Peru. Indoor Air. 2013 Aug;23(4):34252. doi: 10.1111/ina.12027.

64. Helen G, Aguilar-Villalobos M, Adetona O, Cassidy B, Bayer CW, Hendry R, et al. Exposure of Pregnant Women to Cookstove Related Household Air Pollution in Urban and Peri-Urban Trujillo, Peru. Arch Environ Occup Health. 2013 Nov 12. [Epub ahead of print].

65. Commodore AA, Zhang JJ, Chang Y, Hartinger SM, Lanata CF, Mäusezahl $\mathrm{D}$, et al. Concentrations of urinary 8-hydroxy-2'-deoxyguanosine and 8 -isoprostane in women exposed to woodsmoke in a cookstove intervention study in San Marcos, Peru. Environ Int 2013 Oct;60:112-22. doi: 10.1016/j. envint.2013.08.013.

66. Naeher LP. Biomass-fueled intervention stoves in the developing world: potential and challenges. Am J Respir Crit Care Med. 2009 Oct 1;180(7):586-7. doi: 10.1164/rccm.200907-1115ED.

67. Bonjour S, Adair-Rohani H, Wolf J, Bruce NG, Mehta S, Prüss-Ustün A, et al. Solid fuel use for household cooking: country and regional estimates for 1980-2010. Environ Health Perspect. 2013 Jul;121(7):784-90. doi: 10.1289/ ehp.1205987.

68. Painschab MS, Davila-Roman VG, Gilman RH, Vasquez-Villar AD, Pollard SL, Wise RA, et al. Chronic exposure to biomass fuel is associated with increased carotid artery intima-media thickness and a higher prevalence of atherosclerotic plaque. Heart. 2013 Jul;99(14):984-91. doi: 10.1136/heartjnl-2012-303440.

69. Gonzales GF, Tapia V. Birth weight charts for gestational age in 63,620 healthy infants born in Peruvian public hospitals at low and at high altitude. Acta Paediatr. 2009 Mar;98(3):454-8. doi 10.1111/j.1651-2227.2008.01137.x.

70. Intergovernmental Panel on Climate Change (IPCC). 2013. Climate Change 2013: The Physical Science Basis: Working Group I Contribution to the Fifth Assessment Report of the Intergovernmental Panel on Climate Change. Berne: WHO; 2013

71. González C, Paz A, Ferro C. Predicted altitudinal shifts and reduced spatial distribution of Leishmania infantum vector species under climate change scenarios in Colombia. Acta Trop. 2014Jan;129:83-90. doi: 10.1016/j. actatropica.2013.08.014.

72. Alvarez-Falconi PP, Ríos Ruiz BA. Brote de fiebre de Oropuche en Bagazán, San Martín - Perú: Evaluación epidemiológica, manifestaciones gastrointestinales y hemorrágicas. Rev Gastroenterol Peru. 2010 Oct-Dec;30(4):334-40.

73. Bayer AM, Hunter GC, Gilman RH, Cornejo Del Carpio JG, Naquira C, Bern C, et al. Chagas disease, migration and community settlement patterns in Arequipa, Peru. PLoS Negl Trop Dis. 2009 Dec 15;3(12):e567. doi: 10.1371/journal.pntd.0000567.

74. Delgado S, Ernst KC, Pumahuanca ML, Yool SR, Comrie AC, Sterling CR, et al. A country bug in the city: urban infestation by the Chagas disease vector Triatoma infestans in Arequipa, Peru. Int J Health Geogr. 2013 Oct 30;12:48. doi: 10.1186/1476-072X-12-48.

75. McPhaden MJ, Zebiak SE, Glantz $\mathrm{MH}$. ENSO as an integrating concept in earth science. Science. 2006 Dec 15;314(5806):1740-5.

76. Horel JD, Cornejo-Garrido AG. Convection along the coast of northern Peru during 1983: Spatial and temporal variation of clouds and rainfall. Mon Wea Rev. 1986;114(11):2091-2105.

77. Seas C, Miranda J, Gil AI, Leon-Barua R, Patz J, Huq A, Colwell RR, et al. New insights on the emergence of cholera in Latin America during 1991: the Peruvian experience. Am J Trop Med Hyg. 2000 Apr;62(4):513-7.

78. Gil AI, Louis VR, Rivera IN, Lipp E, Huq A, Lanata CF, et al. Occurrence and distribution of Vibrio cholerae in the coastal environment of Peru. Environ Microbiol. 2004 Jul;6(7):699-706.

79. Martinez-Urtaza J, Huapaya B, Gavilan RG, Blanco-Abad V, Ansede-Bermejo J, Cadarso-Suarez C, et al. Emergence of Asiatic Vibrio diseases in South America in phase with El Niño. Epidemiology 2008 Nov;19(6):829-37. doi: 10.1097/ EDE.0b013e3181883d43.

80. Chowell G, Cazelles B, Broutin $\mathrm{H}$, Munayco CV. The influence of geographic and climate factors on the timing of dengue epidemics in Perú, 19942008. BMC Infect Dis. 2011 Jun 8;11:164. doi: 10.1186/1471-2334-11-164.

81. Brunkard JM, Cifuentes E, Rothenberg SJ. Assessing the roles of temperature, precipitation, and ENSO in dengue reemergence on the Texas-Mexico border region. Salud Publica Mex. 2008 MayJun;50(3):227-34.

82. Giraldo A. Primer brote de dengue documentado en la región amazónica del Perú. Bol Of Sanit Panam. 1993;114(6):513-9.

83. Mamani E, García M, Gutiérrez V, Cabezas C, Harris E. Tipificación molecular del virus dengue 3 durante el brote epidémico de dengue clásico en Lima, Perú, 2005.Rev Peru Med Exp Salud Publica. 2005;22(3):161-4.

Correspondencia: Gustavo Gonzales Rengifo Dirección: Av. Honorio Delgado 430, SMP

Teléfono: 3100000 anexo 2535

Correo electrónico:Gustavo.gonzales@upch.pe 